\title{
Experience of the application of remote sensing data in the problems of geo-ecological monitoring of the coal mining area
}

\author{
Olga Giniyatullina, Evgeniy Schastlivtsev, and Vladimir Kovalev \\ Institute of Computational Technologies of SB RAS, Kemerovo, Russia
}

\begin{abstract}
The experience of solving problems of geoecological monitoring of coal mining region with the use of remote sensing data is presented. The results of control over the boundaries of coal-mining enterprises, assessment of the degree of self-growth of dumps, monitoring of the state of vegetation near objects of coal mining and dust load of the area are shown.
\end{abstract}

The current state of the environment in areas with high anthropogenic impact requires constant monitoring by special services and the use of active tools for ecological monitoring. This problem is especially acute in coal-mining regions where as a result of mining fundamental changes in the structure of lands, landscapes, aquatic environments, biological communities, etc. occur. Classical methods of assessing the quality of the environment, for example, sampling and its analysis in the laboratory give the characteristics of objects only at a specific point while real changes can capture large areas that can far exceed the sanitary zones of mining enterprises. The use of remote sensing data to solve a number of problems of geoecological monitoring makes it possible to assess the state of the territories of the region as a whole. It should be noted that in this case laboratory test data become an authoritative source of results confirmation of space images processing, thus ensuring their high reliability.

In Kemerovo Scientific Center of ICT SB RAS for more than 10 years new approaches have been developed for solving geoecological monitoring problems using remote sensing data. The most successful developments that are applied in the work of a number of coal mining enterprises of Kuzbass are presented in this article. In general, remote monitoring is conducted in four directions:

1. determination of the boundaries of disturbed lands;

2. monitoring of reclamation / restoration land process;

3. assessment of the state of biomass in the territory of the facility and outside its sanitary zone;

4. obtaining dust load zones.

Let us provide examples of solving similar problems from each direction.

One of the most urgent tasks is to control the boundaries of licensed coal mining sites or the so-called "problem of black diggers". Violation of official boundaries leads to huge financial losses in the regional budget. It should also be noted that in such places uncontrolled coal mining is being carried out which leads to serious changes in the 
ecological condition of the area as the necessary measures are taken to minimize the negative impact on the environment. There is no work to restore disturbed lands.

In this case, the solution of the problem is to isolate the boundaries of the object under study in high-resolution images (for example, Sentinel $2 \mathrm{~A}$ data of resolution $10 \mathrm{~m} / \mathrm{pix}$ ) and correlate them with the official boundaries of license areas using standard GIS methods (Figure 1).

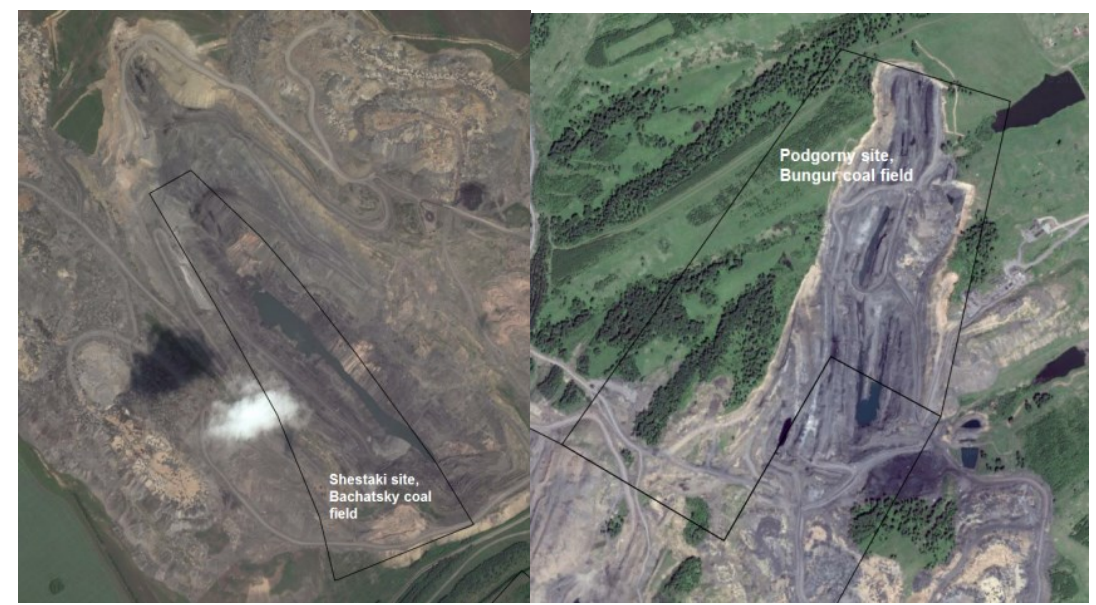

Fig. 1. The control of the boundaries of the coal-mining enterprise (the black line is the boundary of the license area)

As can be seen in Figure 1, the boundaries of both sites are partly outside the range indicated in the mining license. Therefore, an official letter of inquiry is sent to justify the increase in area. It should be noted that for example in this case the increase in the boundaries is justified by the technological need for coal mining in an open way. Transport infrastructure objects and tailing dumps and mine dumps are out of the boundaries, that is why that is not a violation and that enters the zone of the mining lands of coal-mining enterprises.

Another rather complex issue is the remote monitoring of land reclamation process after coal mining. The solution of this problem was described in details by the authors in [1]. The proposed method is based on the processing of a number of images over several years by the SAVI soil index from Landsat 7 data. The most sensitive spectral channel for counting this type of indices is the "extreme red" red-edge band (705-745 nm). The soil indices characterize the degree of coverage of the observed surfaces by vegetation and react well to the minimal presence of biomass on the surface of the object. From our point of view in this case the most convenient way for investigating the interrelated components of "vegetationsoil" is the use of the Gaudrian model. In this model the spherical coefficient of spectral reflection is used: it is assumed that the soil is a Lambert diffuser and the values of the coefficient do not depend on the direction of the sun's rays and sight incidence.

When using the SAVI index to estimate mine dumps the value $\mathrm{L}=1$ was used as objects with no or minimal vegetation expressed in the form of a weak grass cover were researched.

As a result of the processing, the changes in the state of biomass even in minimal amounts are recorded which is characteristic of self-growth processes when the appearance of vegetation is due not to the purposeful formation of the humus layer but to the chaotic transfer of plant seeds by natural processes. Thus, for example, in the study of the mine dump of the coal-mining section for the period from 1992 to 2010 when the work on its formation was actively conducted the indexes were fixed from -0.25 to 0.05 , which 
indicates the appearance of biomass on the surface. Figure 2 shows the results of monitoring the mine dump of the Bachatskiy open-pit (Kemerovo region).

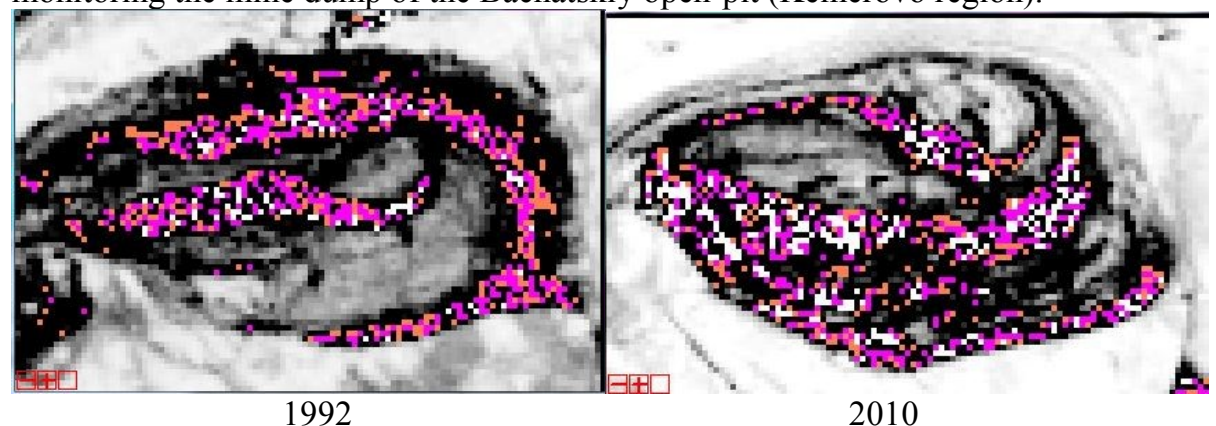

Fig. 2. Results of calculating the soil vegetation index SAVI

As can be seen, the shift of the horizon from the north to the south is clearly traced. The boundary of the soil index passes through 0 , the color markers represent the indices in the range $[0 ; 1]$ which characterize the soil. The indicators that are presented in white color fix inclusions which are an empty rock (i.e/ not a soil fertile layer). As it is clear from the figure the formation of the mine dump went from bottom to top and by 2010 an active process of self-growth of the southern side of the mine dump has been observed. It should be noted that reclamation of land in this mine dump in the specified period of time was not carried out while the average speed of appearance of the first stable manifestations of green mass is 4-5 years. While conducting the research on this method of experimental recultivation sites the index jump is fixed in the second year with its further increase depending on the prevailing species of vegetation.

A rather widespread problem is the estimation of biomass in satellite images using vegetation indices [2]. There are more than 160 different vegetation indices currently. The most common is NDVI (Normalized Difference Vegetation Index). In the course of research of different indices applicability it was proposed to use a combination of indices such as NDVI, Simple Ratio Index (SRI) and Anthocyanin Reflectance Index (ARI) to assess the state of vegetation in coal mining regions. If the first two indices are oriented to the allocation of biomass as a whole then the third index detects the intensity of anthocyanins in the leaves. Moreover, authors suggest to consider these indexes not separately but in combination. As a result, it is possible to obtain integrated vegetation maps based on joint processing of indices (Fig. 3) where the $\mathrm{R}$ band contains the values of the NDVI index, G-SRI and B-ARI.

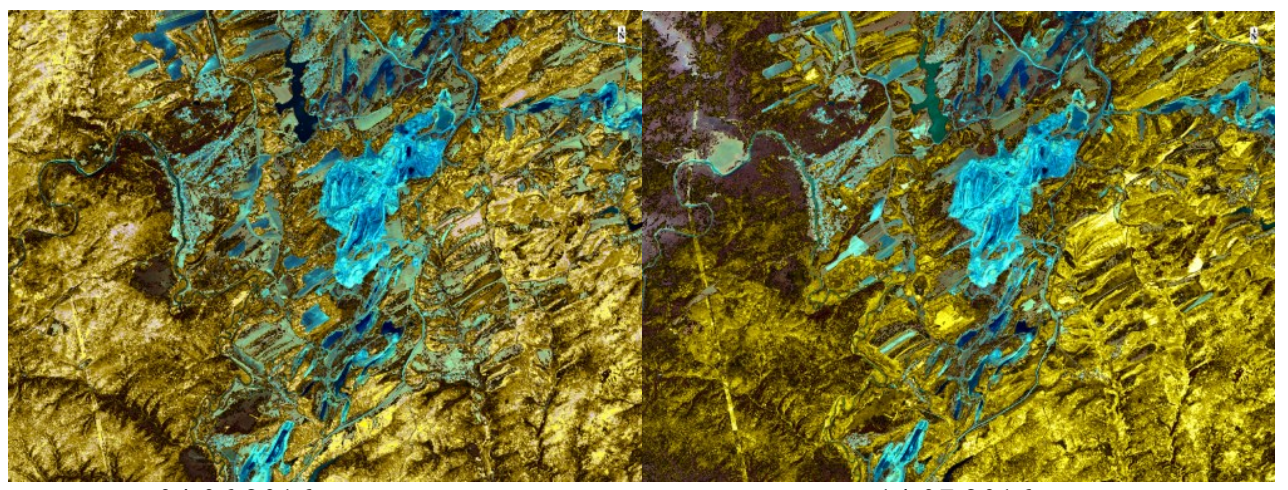

04.06 .2016

14.07.2016 


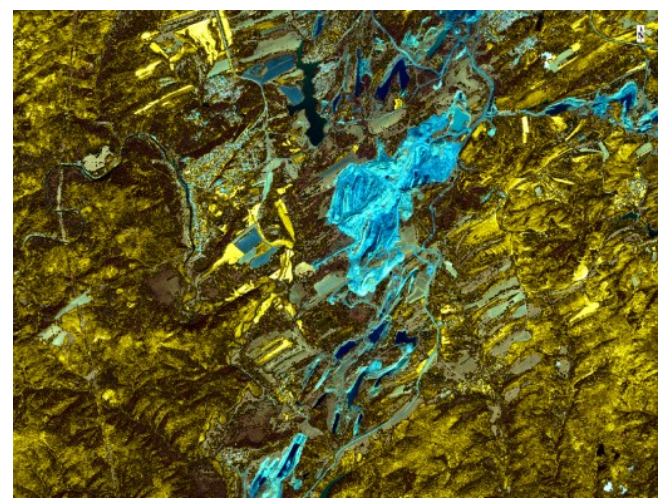

30.08.2016

Fig. 3. The results of calculating the combination of NDVI, SRI and ARI indices

According to the accepted gradation, the normal state of vegetation is estimated in yellow-green tones which corresponds to the biomass of the research region. The presence of brown shades suggests a weakening of vegetation growth. However, as 2016 was very arid then quite large presence of this color is due to natural causes rather than the influence of coal mining. As a rule, weaker vegetation is fixed in the zones of direct contact with coal dump where the biomass is under considerable stress. But, as a rule, these changes are fixed in the zone not exceeding $500 \mathrm{~m}$ which corresponds to the established sanitary norms.

The next important direction of remote monitoring is the estimation of the dust load from the images over the winter period. The estimation is made by studying the intensity of the snow cover near the objects of coal mining. In [3] the authors described in detail the technique for processing images with object-oriented algorithms for selecting segments in an image. The essence of the approach lies in the complex isolation of structural, textural and spatial characteristics of objects in the image according to some rule or their combination. The approach is based on the law of emissions distribution from the source, i.e. the settling of pollutants occurs from the source of pollution with a decrease in concentration at a certain threshold when it is removed. As the maximum deviation in the IR zone is characteristic for snow with increasing pollution then the spectral brightness of its pixels can be determined and it is possible to divide them into some conditional classes in the presence of deviations in the IR spectrum. In fact, the task is to break down the image into segments using as a rule the deviation in the spectral values of the pixel in certain channels, i.e. internal properties of objects.

The best result for determining the distribution of dust on the snow cover is the use of an object-oriented algorithm with the setting of boundaries with varying intensity in any channel and using a complete list of $\lambda$ values when small segments merge into larger ones. As a result, a segmented image of the snow cover is obtained where the boundaries of the change in reflection intensity of the coating are easily identified which characterizes the degree of its contamination.

It should be noted that in this technique the authors carried out a unique research of the confirmation of the results of image processing with calculated values from the ERA software package (www.logos-plus.ru) and data of laboratory researches of snow samples.

Figure 4 shows the results of processing a satellite image of Sentinel 2A using this method with the calculated values of the mathematical model. 


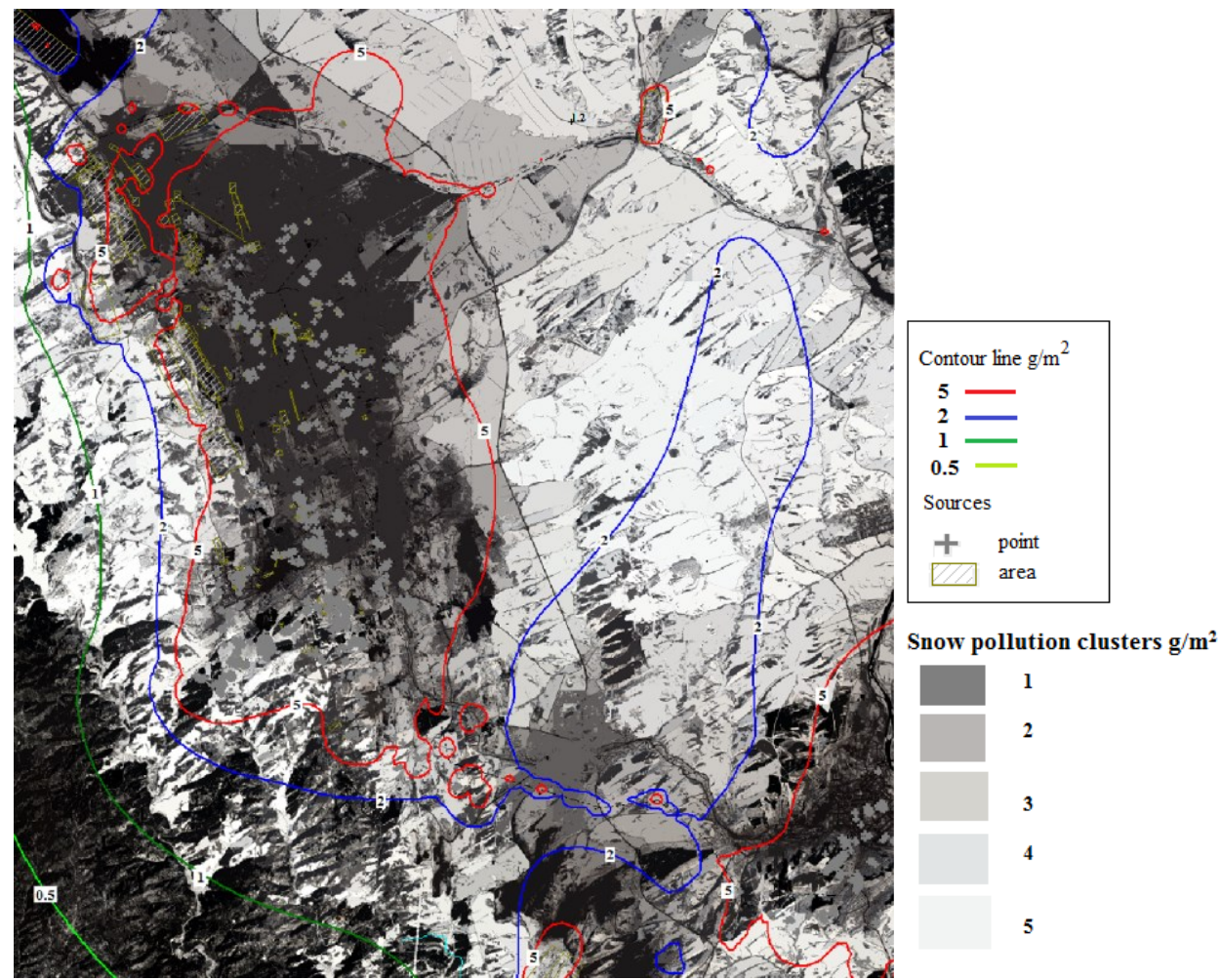

Fig. 4. Combining the results of space images and calculating of the mathematical model

As can be seen, there is a $100 \%$ hit of objects with a sharp drop in the reflection values in the region of the IR spectrum in the contour of the calculated contour line of $5 \mathrm{~g} / \mathrm{m} 2$ (Fig. 4, line 5). The resulting segments correspond to the sources of contamination. After that, the allocated segments are distributed to four more classes with increasing values in the IR spectrum (Fig. 4, lines 1-4) which corresponds to the distribution of contaminators from sources. Thus, it became possible to identify the extent of snow contamination in the officially accepted classification.

As can be seen from the figure, the approach that was developed by the authors to image processing allows to obtain a topical picture of the dust load of the coal-mining region. The thematic products obtained on the basis of this approach make it possible to monitor the dust load of a region with a high technogenic impact throughout the region, and not only at ground control points.

Of course, the above list of tasks is not exhaustive. In this article we have only touched upon the most frequently solved problems of geoecological monitoring using remote sensing data for the coal-mining region. This list is being actively expanded. The emergence of new remote sensing data, methods and approaches to their processing allows creating fundamentally new thematic products and instruments for monitoring the state of the environment.

\section{References}

1. V.N. Oparin, V.P. Potapov, O.L. Giniyatullina, E.L. Shchastlivtsev, Studies into the process of mine waste dump filling up by vegetation using remote sensing data, JMS, Vol. 49, No. 6 (2013). 
2. John G. Lyon, Ding Yuan, Ross S. Lunetta, Chris D. Elvidge, A Change Detection Experiment Using Vegetation Indices, Photogrammetric Engineering \& Remote Sensing, Vol. 64, No. 2 (1998)

3. V. N. Oparin, V. P. Potapov, O. L. Giniyatullina, A. A. Bykov, and E. L. Schastlivtsev, Integrated monitoring of induced air pollution in mining regions, JMS, Vol. 53, No. 5 (2017) 